\title{
Abstracts of the annual scientific meeting of the Association of Clinical Cytogeneticists held at the University of Newcastle upon Tyne on 3 and 4 July 1985
}

\begin{abstract}
Huntington's chorea, G8, and chromosome 4 SANDRA YOUNGMAN, PETER S HARPER, AND DUNCAN SHAW Section of Medical Genetics, University of Wales College of Medicine, Cardiff.
\end{abstract}

A study of eight Huntington's chorea families in South Wales with the DNA probe G8 shows close genetic linkage. Thus the linkage reported in the two original American kindreds appears to apply also to families in South Wales. No definitive example of recombination has so far been detected. In our families, the most likely distance between the marker and disease is $2 \mathrm{cM}$. Such close genetic linkage is of practical importance and is the first step in finding the primary gene defect. The DNA probe G8 maps by in situ hybridisation to the short arm of chromosome 4 , but if the gene is to be more accurately mapped more short arm chromosome 4 markers must be isolated. Future work will involve the isolation of chromosome 4 probes from a cell hybrid containing a rodent cell background and only human chromosome 4 . Probes which map to the short arm will be required; therefore, there is a need for a panel of chromosome 4 translocations involving the short arm and chromosomes for which selection can be applied (X and 17). Cytogenetics units are especially well placed to recognise and contribute this important material.

Rapid karyotyping of chorionic villus cultures by a single cell harvest technique

U CLAUSSEN, D E HEATON, AND D $\mathrm{v}$ COLEMAN

Cytogenetics Department, St Mary's Hospital Medical School, London.

Chromosome preparations can be made from chorionic villi either directly or by tissue culture. Chromosome morphology and $G$ banding resolution of cultured cells tend to be of a consistently higher quality than that obtained from direct preparations, but the 10 days or more necessary for culture is regarded as a disadvantage of this technique. Using the 'pipette method' developed by Claussen (1980) for harvesting cell cultures, we have obtained good quality $\mathrm{G}$ banded metaphase spreads from trophoblast cultures in five to seven days. Individual mitoses were removed from coverslip cultures (Heaton $e t$ al, 1984) by means of a siliconised, attenuated pasteur pipette (bore 20 to $60 \mu$ ) held in a simple micromanipulator attached to an inverted microscope $(\times 63)$. The cells were expelled into $0.075 \mathrm{~mol} / \mathrm{KCl}$. The swollen cells were then taken back into the pipette into which methanol/acetic acid (3:1) was rapidly drawn. Fixed cells were then dropped onto cleaned slides. Cultures can be maintained for further procedures. This approach, using short term culture, thus confers advantages over both direct preparation and long term cultures.
Supernumerary markers derived from chromosome 15 associated with trisomy 21

TONY ANDREWS AND MARK McKINLEY

Wessex Regional Cytogenetics Unit, The General Hospital, Salisbury.

Cytogenetic analysis using a range of banding techniques including GTL, Ag-NOR, CBG, QFQ, and DA/DAPI, was used to identify the origin of a supernumerary marker in two cases of atypical Down's syndrome. Case 1, the second child of healthy parents (mother 39, father 41 years), was referred for cytogenetic studies at 11 years. She was clinically diagnosed as having Down's syndrome with some atypical features including a total lack of language development, behavioural problems, and tonic-clonic and myoclonic seizures. Both parents had normal karyotypes. The karyotype of the proband was interpreted as being $48, \mathrm{XX},+\operatorname{dic}(15)(\mathrm{pter} \rightarrow \mathrm{q} 12$ or $\mathrm{q} 13: \mathrm{q} 12$ or $\mathrm{q} 11 \rightarrow \mathrm{pter})$, +21 . Case 2, a male newborn, was the fifth child of healthy parents (maternal and paternal ages 24 and 27 years respectively). The pregnancy had been complicated by mild polyhydramnios. A clinical diagnosis of Down's syndrome was made soon after birth. The child was also noted to have brachycephaly, micrognathia, and a small mouth with narrow palate. Apnoeic attacks, brachycardia, and convulsions became progressively more severe until death at 33 days. Necropsy revealed an atrial septal defect but no other gross abnormalities. Both parental karyotypes were normal. The karyotype in this infant was 48,XY, $+\operatorname{dic}(15)($ pter $\rightarrow q 12$ or $q 13:: q 13$ or $q 11 \rightarrow$ pter $),+21$. We propose the term $+\operatorname{dic}(15)$ rather than $+\operatorname{inv} \operatorname{dup}(15)$ as a more appropriate description of these supernumerary markers.

Molecular genetics of Duchenne muscular dystrophy and $\mathrm{X}$ linked mental retardation

KAY E DAVIES, HUW DORKINS, TERENCE SMITH, SUSAN KENWRICK, AND SARAH BALL

Nuffield Department of Clinical Medicine, John Radcliffe Hospital, Oxford.

The molecular analysis of genetic diseases where the underlying biochemical defect is known is now straightforward in most instances. The gene sequence in which the mutation has occurred can be cloned as by mRNA enrichment, by differential screening between normals and patients, or by the synthesis of a DNA oligonucleotide probe directly from the protein sequence. For genetic diseases where the underlying molecular defect is not understood, the mutation can be identified by 'reverse genetics'. The mutation is first localised to a chromosomal region by linkage analysis with restriction fragment length polymorphisms (RFLPs). The use of DNA markers in the 
diagnosis of Duchenne muscular dystrophy and $\mathrm{X}$ linked mental retardation (fragile $\mathrm{X}$ ) will be discussed.

Two Y chromosome abnormalities investigated by cytogenetic and DNA studies

S J IMRIE, D H GILMORE, R A L LOW, N AFFARA, D JAMIESON, L FLORETIN, J CROSSLEY, A CAINE, AND E BOYD

Department of Medical Genetics, Royal Hospital for Sick Children, Glasgow.

Two subjects with an abnormality of the $Y$ chromosome are described. The first is a $48, \mathrm{XX}, \mathrm{i}(\mathrm{Yq}) \mathrm{i}(\mathrm{Yq})$ karyotype found in a female aged 16 years investigated cytogenetically because of primary amenorrhoea. The second, found in a female referred for recurrent miscarriage, is an apparent deletion of the short arm of the Y. In both cases additional studies were carried out using DNA probes known to map to the long or short arm of the Y.

Studies of Down's syndrome using chromosome 21 specific DNA probes

JAMES GALT

Department of Medical Genetics, Royal Hospital for Sick Children, Glasgow.

A number of single copy DNA clones have been isolated from a chromosome library enriched for chromosome 21 by flow sorting. Five of these have so far been mapped to chromosome 21 using a somatic cell hybrid. Regional mapping of these probes, and of another probe previously assigned to chromosome 21 , was carried out using another somatic cell hybrid and also sorted DNA. Three probes were shown to map on either side of the breakpoint $21 q 21 \cdot 2$. Of these probes, four have now been shown to detect restriction fragment length polymorphisms (RFLPs) and are currently being used with some success to determine the parental origin of non-disjunction in families with Down's syndrome. Several other probes have recently been isolated from the same library using a different method of recombinant selection. These are about to be mapped and then tested for RFLPs. All the probes isolated will also be tested for expression, as they might contain genes important in the pathogenesis of Down's syndrome.

Marking time with methotrexate

JOHN R K SAVAGE AND R PRASAD

Cytogenetics Section, MRC Radiology Unit, Harwell.

According to current ideas, methotrexate (MT) either blocks cells in $\mathrm{G} 1$ before they enter $S$ phase or at a particular stage within $S$. In either case, cells accumulate at the block and can be released by supplying thymidine or a thymidine analogue. This is the basis for using MT for cell synchronisation. If release is accomplished by supplying bromodeoxyuridine, chromosomes at subsequent metaphase can be stained for replication banding and the pattern they carry should indicate where the block was. If pre-S there will be no replication bands, but if mid-S, TT bands will be present characteristic of the subphase reached before the block operates. Primary Syrian hamster fibroblasts were exposed to MT for 16 hours then released with medium containing BrdU at $20 \mu \mathrm{g} / \mathrm{ml}$ and the mitotic wave sampled at two hour intervals. The first experiment used one year old Sigma MY at concentrations from $10^{-6}$ to $10^{-4}$. This had no effect on the FDM or $S$ phase progression compared with the untreated controls. The second experiment employed new Sigma and new Lederle MT at $10^{-6} \mathrm{~mol} / \mathrm{l}$. The mitotic index suggested some blocking and partial synchronisation, but all subphases of $S$ were present on release in proportions almost unchanged from control cultures. We concluded: (1) that MT can lose efficacy on storage; (2) that in these fibroblasts there has been no specific blocking point in the cycle, but an overall slowing down and marking time which speeds up again without confusion when the folate antagonist is removed.

\section{$S$ phase progression in low folate medium}

R S DEWDNEY, J R K SAVAGE, AND M FITCHETT

Cytogenetics Section, MRC Radiology Unit, Harwell; and Wessex Regional Cytogenetics Unit, The General Hospital, Salisbury.

A low folate version of Iscove's modification of Dulbecco's MEM is now widely used to promote the expression of fragile chromosome sites and also as a means of obtaining generally lengthened chromosomes in lymphocytes to improve band resolution. We have used the facility of $S$ phase subdivision by replication banding (Savage and Prasad, J Med Genet 1984;21:204) to determine the profiles of S phase progression of stimulated normal male lymphocytes in low folate and control media. The early subphases of $S$, when the pale $G$ bands (thought to contain the genetically more important DNA) replicate, are markedly lengthened in low folate conditions, whereas the remainder of $\mathrm{S}$ phase appears to proceed at a rate similar to that observed in control medium.

Are some modern cytogenetic techniques the cause of more bother than they are worth?

E.LIZABETH GRACE

Cytogenetics Department, Royal Hospital for Sick Children, Edinburgh.

Analysis of chromosome banding patterns is impaired when chromosomes are relatively contracted and the number of bands and the mitotic index are low. However, modern cytogenetic techniques, designed to improve the overall quality of preparations, can often cause worse problems of interpretation, uncovering small differences in banding patterns between homologues, or alternatively inducing increased levels of gaps, breaks, fragments, and structural rearrangements. Examples include minor differences in 11p, 17p, 6q, and 19p, and up to $25 \%$ breakage at consistent sites not normally regarded as fragile. As yet it is almost impossible to assess the significance, if any, of such observations, especially if the patient is a dysmorphic child or a stillbirth/neonatal death. Family studies are often unhelpful and are also time consuming. Thus, it is important to establish the 'normal' karyotype as far as is possible in the light of new technology and to this end a collaborative venture among interested laboratories may be helpful. 


\section{Cytogenetic survey of an institution for the mentally retarded}

C J ENGLISH, E V DAVISON, M BHATE, AND L BARRETT Department of Human Genetics, University of Newcastle, Newcastle upon Tyne.

A cytogenetic survey of males in a north-eastern institution for the mentally retarded is in progress, primarily to detect fragile X males. Preliminary results show that of 360 investigated, $40(11.1 \%)$ have Down's syndrome, 29 $(8.1 \%)$ express the fragile $X$ chromosome, and $17(4.7 \%)$ have other chromosome anomalies. Additionally, four autosomal fragile sites were detected in a further seven men. Investigations of the 29 fragile $X$ males showed no correlation between the level of fragile $X$ expression and either age or degree of mental retardation. In eight female relatives (three retarded, one 'dull', four normal) there was a correlation between level of fragile $X$ expression and age $(r=-0.791, d f=7, p<0 \cdot 02)$. Late replicating $X$ studies of two retarded and one 'dull' female showed the latter to have the lowest proportion of active fragile $\mathrm{X}$ chromosomes. Clinical investigations of the fragile $\mathrm{X}$ males showed 24 of 25 to have macro-orchidism and 16 of 22 appear to have long narrow facies. An anthropometric study is being carried out to establish facial shape by actual measurements. The 29 fragile $\mathrm{X}$ males belong to 26 pedigrees and further information has been obtained for 10. In only three pedigrees was classic $X$ linked inheritance observed. Four had affected females, two probands were isolated cases, and one pedigree exhibited transmission through apparently normal males. These 10 families included another 16 mentally retarded males, six mentally retarded females, and 67 possible female carriers of whom 26 are at, or below, reproductive age.

\section{A classification of three break structural rearrangements R T HOWELL \\ South-Western Regional Cytogenetics Centre, Southmead Hospital, Bristol.}

The majority of structural chromosome rearrangements encountered in clinical cytogenetics originate from one or two breakpoints, but a smaller proportion can be explained only by including a third breakpoint. The range of possible structures formed by three break rearrangements is determined by the distribution of the breaks on one, two, or three chromosomes; the positions of the breakpoints with respect to the centromere(s) of the participant chromosome(s); and the pattern of rejoining of the six broken ends. It is possible to distinguish 20 different stable monocentric rearrangements which include not only the familiar insertions and the three way translocation but also double inversions, combinations of inversion and translocation, combinations of inversion or translocation with interstitial deletion, and translocation with a ring chromosome. Examples of some of these types ascertained in the laboratory include the prenatal diagnosis of a pericentric inversion and interstitial deletion of chromosome 18; ring $\mathrm{X}$ and $\mathrm{X} ; 11$ translocation in a girl with Turner's syndrome (M A Hamill, personal communication); and pericentric inversion of chromosome 1 and a $1 ; 17$ reciprocal translocation in bone marrow from a preleukaemic patient.
Evaluation of Magiscan II: an interim report

M DAKER

Paediatric Research Unit, Guy's Hospital Medical School, London.

Two aspects of the evaluation were discussed in outline in order to convey some idea as to the potential of Magiscan in the diagnostic analysis of blood culture chromosomes. The first study was related to a series of 45 chromosome abnormalities that were analysed blind. In two cases the abnormality was missed. The first, 46,XYdel(4)(p15.02), was, however, from poor spreads, while the second, $46, \mathrm{XY}, \mathrm{t}(9 ; 17)(\mathrm{q} 22 ; \mathrm{q} 23)$, had been very difficult to detect using conventional microscopy. In all other cases (excluding mosaics) at least some indication of the abnormality was apparent from the Magiscan karyotypes, although it was generally difficult to be precise about breakpoints in structural changes. In the second study, diagnostic chromosome analysis, using Magiscan, was carried out on blood cultures from 56 patients. Seven abnormalities were detected. All were numerical chromosome anomalies, except for one balanced Robertsonian translocation involving chromosomes 13 and 14. All the results were subsequently checked by conventional analysis, and the only discrepancy found involved a mosaic situation where, unexpectedly, only the minor cell line $(45, X)$ was detected. The major cell line $46, \mathrm{X}, \mathrm{i}(\mathrm{Xq})$, present in three-quarters of cells, was not observed on the Magiscan analysis. The importance of the latest software for straightening prophase chromosomes was also discussed. The facility for storing images of specific straightforward chromosomes, which can later be recalled side by side on the screen, was seen as having considerable potential for analysing prometaphase or prophase chromosomes from subjects with very small deletions.

Prenatal diagnosis by chorionic villus sampling: cytogenetics results

M E FERGUSON-SMITH, C FREW, J MCIVER, D GILMORE, R CHATFIELD, M WHITTLE, V HOOD, AND A CAMERON Department of Medical Genetics, Royal Hospital for Sick Children, Glasgow.

The option of prenatal diagnosis by CVS is now being offered in the west of Scotland. Fetal karyotyping from chorionic villi can be achieved (with $99 \%$ success) using a modified 'direct' method. The mitotic index depends on the quality rather than the quantity of the tissue. Chromosomes suitable for analysis have been obtained from as little as $<5 \mathrm{mg}$. An average $20 \mathrm{mg}$ sample can yield sufficient material for additional DNA or enzyme analysis. Forty-two diagnostic cases were referred and samples were obtained in 37. Chromosome studies were successful in all of these. Fourteen normal babies were delivered, as predicted, and 16 pregnancies are continuing. There were three terminations. Of the four spontaneous abortions, one occurred after a breast biopsy under general anaesthetic four weeks after CVS and three aborted within seven days of CVS. One of these underwent the procedure because of maternal age. Of the 15 candidates with maternal age indication, three had non-viable fetuses on ultrasound before CVS. Maternal age should be considered as a separate category when evaluating the risks of CVS procedure. Mosaicism, which may occur in placental tissues, should be checked by amniocentesis to confirm the 'true' fetal karyotype. 